\title{
Espacenet, Patentscope and Depatisnet: a comparison approach
}

\author{
Authors: \\ Jürgens, Björn 1', Herrero-Solana, Victor ${ }^{2}$ \\ ${ }^{1}$ CITPIA PATLIB Centre, \\ Agency of Innovation and Development of Andalusia \\ Seville, Spain \\ E-Mail: bjurgens@agenciaidea.es \\ ${ }^{2}$ SCImago-UGR (SEJ036) \\ Universidad de Granada \\ Granada, Spain \\ E-Mail: victorhs@ugr.es
}

\begin{abstract}
Espacenet, Patentscope and Depatisnet are known as the main multinational patent databases offered by patent authorities which are available to the public free of charge. As all three systems have substantially improved in the last few years, a comparison of their functionalities and capabilities, as discussed herein, is useful for those unfamiliar with the recent developments. In order to present the comparison, the following aspects were analysed: data coverage, search functionality, result list, bibliographic view of records and patent data export options. Case studies are presented where the search systems were compared in the field of nanotechnology. The analysis concludes that Espacenet has the best features for searching, Patentscope the best for analysis and Depatisnet the best for complex search tasks.
\end{abstract}

\section{Keywords:}

Patent Database; Comparison; Features; Functionalities; Free of charge; Open Access

\section{Introduction}

Online patent search systems have evolved considerably over the last two decades; from cryptic 'text only' command-line databases accessible via modem dial-up on a costly pay per record basis, to today's sophisticated web-based search systems accessible via the Internet which are often free of charge. These free databases made patent information popular to a wider audience and have substantially improved over the last few years, now offering patent search functionalities and additional features which were previously only available from commercial providers.

When it comes to understanding more about the advantages and disadvantages of patent databases, few studies are available which compare these databases. Many studies have become outdated or do not include a description of sources that are free of charge. Smith [1] compared online host patent databases available in the late eighties, Lambert [2] compared online host databases and the upcoming Internet patent databases in the late nineties and Schwander [3] evaluated patent searching resources comparing professional and free online databases in 2000. The more recent studies are from Stock [4] and González \& Zuleta [5], both of which compared some commercial providers with various free providers. None of the studies focused on free of charge sources or gave a direct comparison of their features and functionalities.

Regarding free patent and open access sources, Espacenet, from the European Patent Office (EPO), Patentscope from the World Intellectual Property Organization (WIPO) and Depatisnet from the German Patent and Trademark Office (DPMA) are all patent search systems offered by major patent authorities which do not only cover their own patent collection, but also collections from a multitude of countries- making them one of the most popular free of charge patent searching tools available. 
Although there are many similarities between these three products, it is of interest to know more about each database in order to choose the right one for the purpose of the patent search. In this study we analysed these patent search systems, taking into account all of their new features and functionalities which were added in recent years and directly compared them according to four key aspects outlined in section 2 .

The study was completed in the framework of a study about nanotechnology patents in Spain (see Acknowledgements section), in order to find out more about the strengths and weaknesses of these patent search systems and to evaluate their potential use for this study.

\section{Material and Methods}

Espacenet, Patentscope and Depatisnet are all accessible via links on the homepage of their corresponding patent authorities (www.epo.org ; www.wipo.int and www.dpma.de ). Furthermore they can be accessed via their direct webpages, Espacenet being the only one with its own domain (Espacenet.com) whereas Patentscope and Depatisnet are accessible on subdomains of the patent authority's homepages (http://patentscope.wipo.int and https://depatisnet.dpma.de/DepatisNet).

Regarding the analysis methods used to compare all three patent searching systems, a direct comparison was made during the period of July-September 2014 by taking into account their functionalities and features

The following aspects were analysed:

- Data coverage

- Search functionality

- Result list of records

- Bibliographic view

- Patent data export

This direct comparison makes it easier to see the differences of each product and helps a user to understand the special features of each analysed patent search system.

\section{Results}

\subsection{Data Coverage Comparison}

Before starting a patent search it is crucial to know the country coverage of the database, since it is of no use if a database has a good search feature but does not offer coverage for the country the searcher is interested in. Therefore, first of all, the patent data coverage of Espacenet, Patentscope and Depatisnet was analysed. The data for this analysis was extracted from dedicated webpages provided by the corresponding patent authorities containing statistical information about the databases [6] [7] [8].

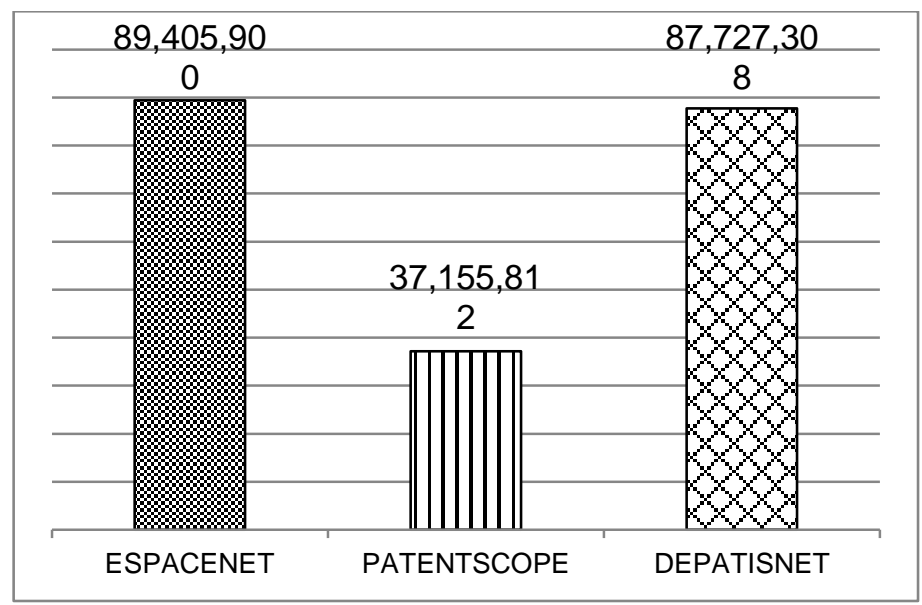

Fig. 1: Number of available patent records in total 
As seen in Fig.1 both Espacenet Worldwide Database and Depatisnet currently covers nearly 90 million patent documents, which makes them the two free of charge patent databases with the largest coverage. Patentscope has a substantially lower coverage with 37 million documents, but according to WIPO [9] the record counting in the Patentscope statistics is one record per invention and not per publication (e.g. patent application and patent grant) so we estimate that the comparable figures of Patentscope are $30 \%$ higher. Patentscope therefore covers approximately 50 million documents, still significantly less than Espacenet and Depatisnet.

When it comes to the coverage per countries we analysed a sample including some major patenting countries and authorities (CN, JP, US, DE, EP, WO) and the patent collection of Spain (Fig. 2). Once again Espacenet and Depatisnet showed similar coverage levels in the main patent collections (WO, EP, US and JP).The German collection in Patentscope was not available at the time of the study, but was to be added by the end of 2014 [10]. It is surprising that Espacenet shows slightly more German patent document records than in Depatisnet, since one would assume that the database from the German Patent Office has the most complete German collection. This finding had no evident explanation and could be explored in a more in-depth study about coverage and counting methods but would exceed the scope of this article.

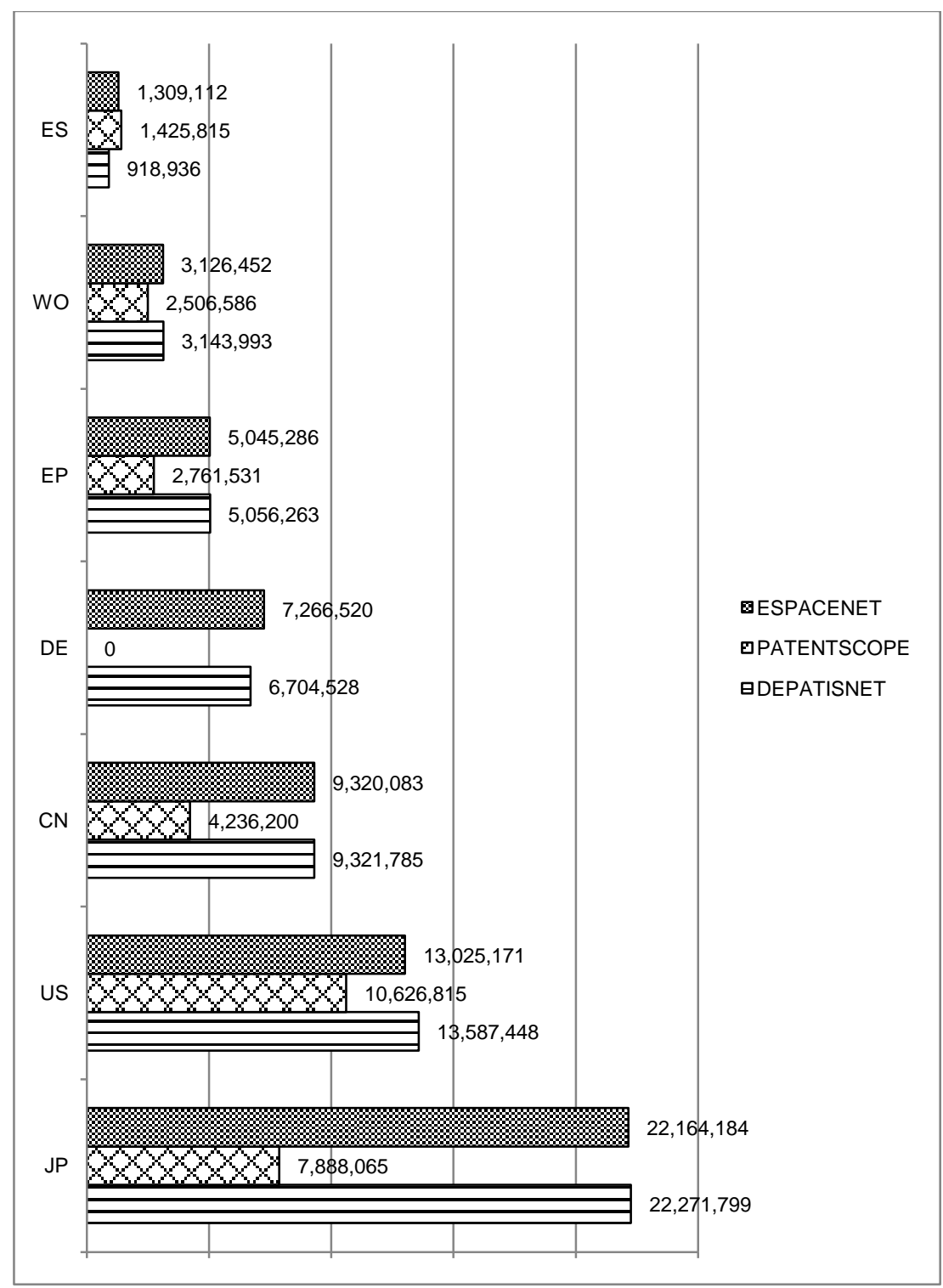

Fig. 2: Data Coverage Comparison per selected countries

Regarding the number of countries or the number of patent office collections per database a similar result is given: both Espacenet and Depatisnet have more than one hundred patent collections (country + WO and EP collections) - figures which more than double Patentscope's total (Fig. 3). This also explains the much higher number of patent documents in their databases. 


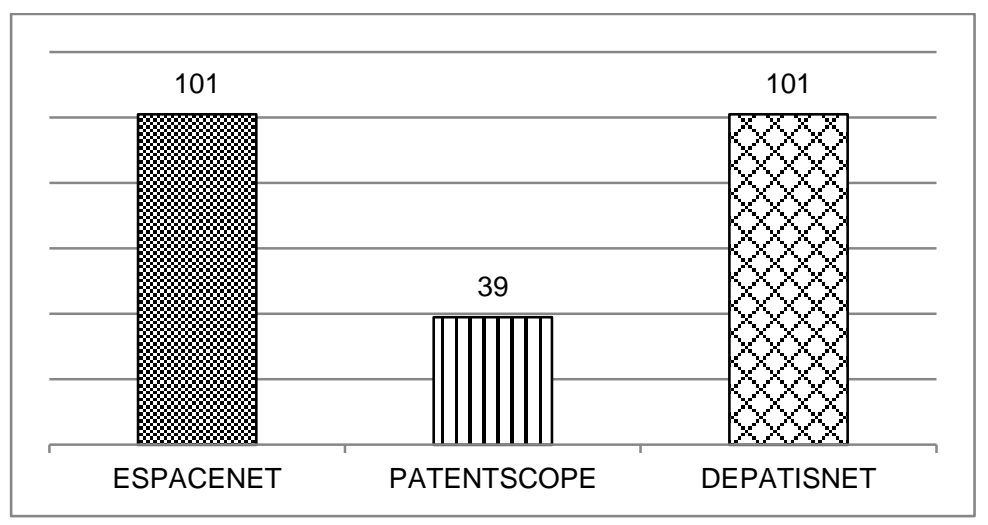

Fig. 3: Number of available patent collections in total (patent authorities)

Full text search can be an important aspect for a patent searcher, since sometimes the features being searched may not at all appear in the title and abstract. In this case the comparison is in favour of Patentscope due to an ongoing effort of WIPO to digitalize country patent collections via optical character recognition (OCR). Although this technology is not always accurate and may lead to text recognition errors, thanks to this initiative, Patentscope can provide 19 patent collections with full text searching capability, whereas Espacenet offers full text search only for EP and WO documents and Depatisnet for DE patents (Fig. 4).

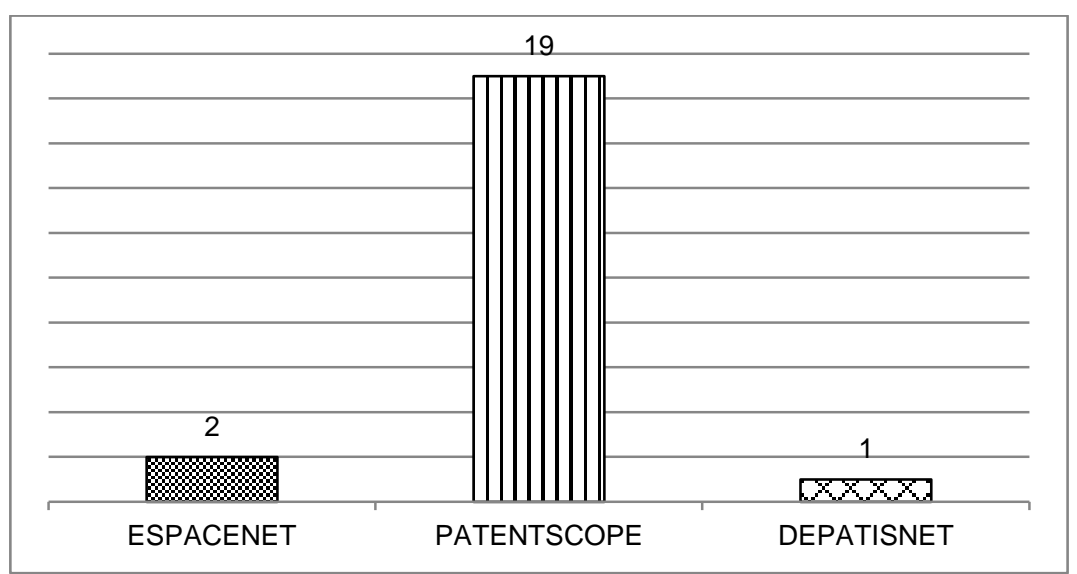

Fig. 4: Number of patent collections with full text being searchable

\subsection{Search Functionalities}

After the patent coverage, the second most important aspect in comparing the search functionalities of each database is the search interface, as it determines whether complex patent searches can be conducted. To have a better understanding of this aspect, we first took a closer look at the search interfaces available in each database and what characteristics they offered to the patent searcher.

In patent databases one can distinguish between number search, form search and command line search interfaces. As we can see in Table 1, all three databases offer these types of interfaces to the users, although the search interface names can differ quite substantially and in some cases could confuse the user e.g. the form search in Espacenet is called "Advanced Search" whereas the same type of search interface in Depatisnet is called "Beginner search". 


\section{Command line searching \& search fields}

Espacenet's Smart Search interface is a "Google style" single entry multi-search field. The function is named "smart", because the search engine tries to automatically recognize the type of search field corresponding to each search term. For example if "Bayer 1999" was entered, the system will identify the German company Bayer as an applicant or inventor and 1999 as a publishing year.

In addition, Espacenet's Smart Search allows command line searches using operators and field identifiers [11], but many users are not aware of this functionality. As shown in the example in Fig. 5 operators and field identifiers were used to retrieve nanotechnology-related patents with Bayer as an applicant, the keywords "nano" and "tube" in the abstract and 2010 as publication year.

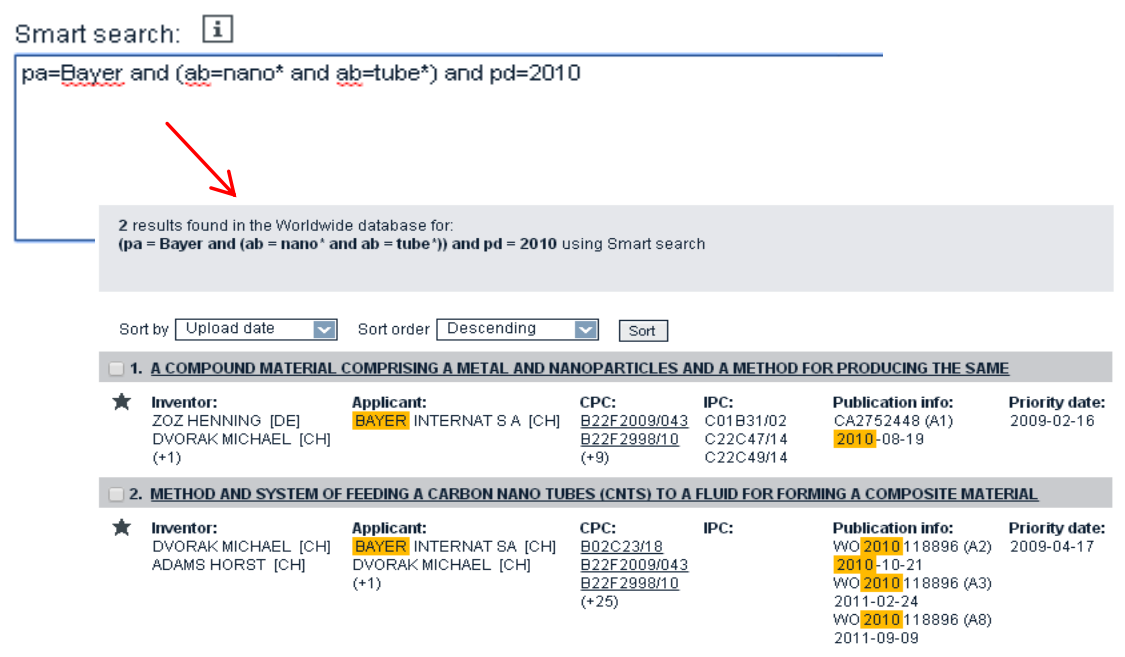

Fig. 5: Command line search with "Smart Search"mode (Screenshots from Espacenet)

When it comes to the number of available search fields in each of the available search interfaces, Patentscope provides the most powerful search interface with 51 search fields available [4], followed by 36 in the command line search of Depatisnet (Expert and Ikofax search) [5], and 16 in Espacenet's Smart Search [6].

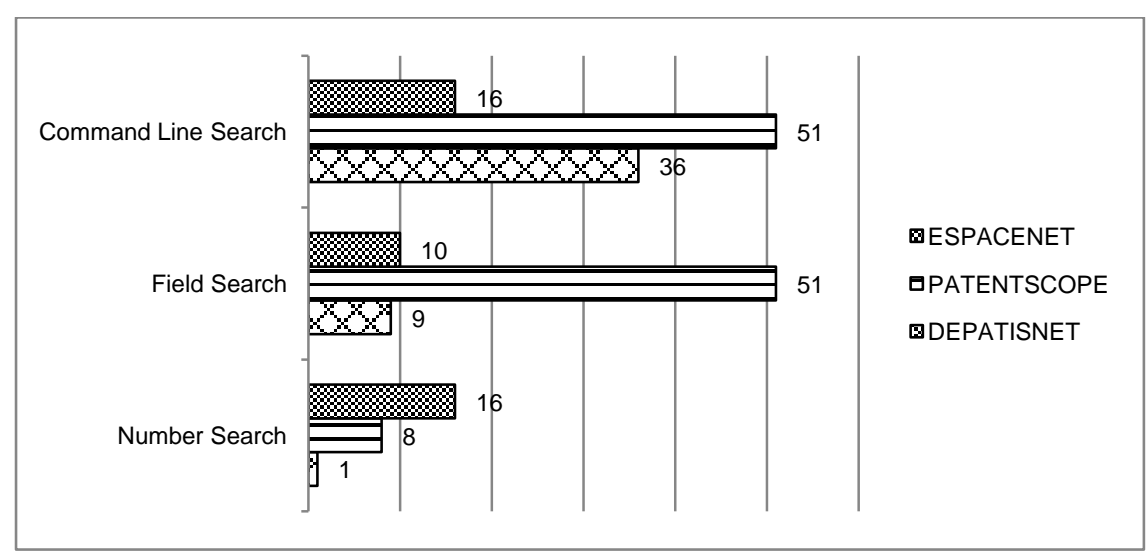

Fig. 6: Number of available Fields in each Search Interface 


\section{Number search}

The approach taken to enable number searching in patent databases is another important aspect to consider, since unfortunately the syntax of patent publication numbers is not always the same and can differ from database to database. Compounding this problem is the fact that publication numbers can vary from one country to another.

For the comparison of how the number search engines were able to handle this problem we took a PCT patent application from a nanotechnology-related patent publication entitled "Method and system of feeding a carbon nano tubes to a fluid for forming a composite material", published in the name of Bayer International with its corresponding international publication number "WO 2010/118896 A2" as highlighted in Fig. 7. We checked how the compared search systems were able to retrieve the document using several different possible syntax variations of the publication number.

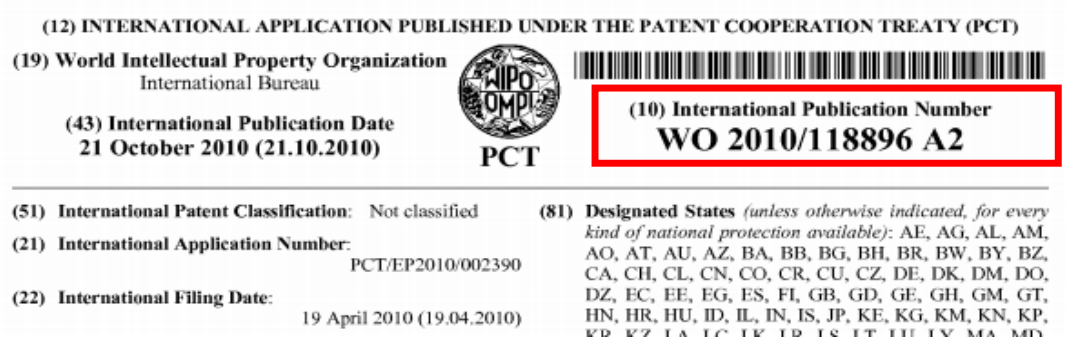

Fig. 7: Header of an original PCT Patent Application

Initially, we used the exact spelling as stated in the original PCT document with slash and spaces, assuming that this is the spelling a non-expert user would use to look up this document in a patent database. Furthermore we checked the number search capabilities of the patent search systems with simpler spelling variations (without slash, without spaces and without kind codes).

To our surprise, Espacenet and especially Patentscope had problems retrieving the document with most of the spelling variations shown in Table 2 .

\begin{tabular}{|r|c|c|c|}
\hline Syntax used & ESPACENET & PATENTSCOPE & DEPATISNET \\
\hline WO 2010/118896 A2 & Not retrieved & Not retrieved & Retrieved \\
\hline WO 2010118896 A & Not retrieved & Not retrieved & Retrieved \\
\hline WO2010118896A & Retrieved & Not retrieved & Retrieved \\
\hline WO2010118896 & Retrieved & Retrieved & Retrieved \\
\hline
\end{tabular}

Table 2: Number Search Case Study Results

Espacenet's number search engine was not very flexible when it came to interpreting different spelling variations because no results were obtained when using the PCT patent number with its slash symbol. Further, by just using space symbols, the search led to incorrect results, because Smart Search misinterpreted the kind code as a classification symbol and thus led to another patent (Fig.8). 
In this comparison only the number search of Depatisnet via its Family search interface was able to retrieve the WO Document with all the different syntaxes and in our experience was the most flexible and successful database when it came to searching by patent publication numbers in general.

\section{Operators \& Wildcards}

When we analyzed the operators available for patent searching across the three databases, our study revealed nearly the same functionalities (Table 3), with only one exception. Depatisnet is the only database that offers left truncation. Left truncation can be very useful for certain types of searches and is a powerful search option not available in many databases in general since it needs a special treatment of the indexed data.

\begin{tabular}{|l|r|c|c|c|}
\cline { 2 - 4 } \multicolumn{1}{c|}{} & ESPACENET & PATENTSCOPE & DEPATISNET \\
\hline \multirow{3}{*}{ Boolean Operators } & AND & Yes & Yes & Yes \\
\cline { 2 - 5 } & OR & Yes & Yes & Yes \\
\cline { 2 - 5 } & NOT & Yes & Yes & Yes \\
\cline { 2 - 5 } & Any length & Yes & Yes & Yes \\
\cline { 2 - 5 } & Precisely 1 character & Yes & Yes & Yes \\
\cline { 2 - 5 } & 1 or no characters & Yes & Yes & Yes \\
\cline { 2 - 5 } & Right truncation & Yes & Yes & Yes \\
\cline { 2 - 5 } & Left truncation & No & No & Yes \\
\hline \multirow{2}{*}{ Limitations } & Proximity operators & Yes & Yes & Yes \\
\cline { 2 - 5 } & Max. search terms per field & Yes (10) & No & No \\
\cline { 2 - 5 } & Max. search terms per mask & Yes (20) & No & No \\
\hline
\end{tabular}

Table 3: Available Operators \& Wildcards

Regarding the maximum search terms allowed per field and per mask it is important to point out one of the main limitations of Espacenet is that the interface only allows a maximum number of 10 search terms per field and 20 per mask. For most simple searches this is not a problem and therefore many users may not notice the limitation, but for patent professionals this is one of the main disadvantages of Espacenet compared to the other two databases, especially when facing more complex searches. 


\section{Classifications}

Patent classifications can be very helpful for effective patent searching and apart from the standard international patent classification (IPC), the more detailed cooperative patent classification (CPC) is a very powerful tool, which is why we included both the IPC and the CPC in our comparison.

As expected, all three databases support IPC, both as a search field in the interface and also offering access to the classification so the user can browse and lookup relevant classifications. When it comes to CPC things look different, in Patentscope CPC is not searchable and browsable, whereas in Depatisnet CPC is only searchable in their command line interface mode Ikofax (although CPC support is not mentioned on the Depatisnet website). Regarding Espacenet, CPC is fully supported as the EPO administers the CPC. Espacenet offers powerful browsing and searching function of the $\mathrm{CPC}$ and in our experience is a very positive feature of this patent search system. Furthermore the direct integration of $\mathrm{CPC}$ as a popup in the result lists turned out to be very useful, because it allows a quick check of the classification without having to leave the patent search interface.

\section{Other Search Features}

Besides search interfaces, operators and classification support, we also identified other search functionalities, which in our opinion, are very useful and can be compared between the databases (Table 4). One example is the dedicated Saving Search Queries feature, which was only offered by Patentscope in this comparison, although in Espacenet the user can save launched searches by bookmarking the search results webpage. Another feature is the Search History, not offered in Depatisnet, but offered by Espacenet and Patentscope, although the Search History function in these databases only lets the user view a list of their executed search commands and does not allow users to combine the search steps with Boolean operators (a feature provided in most commercial databases).Both, Search Queries and Search History, are saved by Patentscope on its server, which requires a (free) user registration. Espacenet does not offer user registration and saves the search history data locally on the computer which has the advantage that no previous registration has to be done, but the disadvantage that the data is lost once the computer is switched or the browser cache data is deleted (see also section3.5 below).

A further feature is the RSS support offered by Espacenet and Patentscope. RSS is a web technology which uses web feed formats to enable publishers to syndicate automatically frequently updated information (like news, blogs, etc.) eliminating the need for a user to manually check the website for new content. Instead, their browser (or alternatively specific RSS reader software) constantly monitors the site and informs the user of any updates.

In the case of Espacenet and Patentscope RSS feeds can be generated for each patent search query, the feed being updated for every new patent, which matches the search criteria and has been added to the patent database. This, in our opinion, is a very useful but underrated feature, since it can be used to create a low cost patent monitoring and technology watch tool.

ESPACENET PATENTSCOPE DEPATISNET

\begin{tabular}{|r|c|c|c|}
\hline Saving patent lists & Yes & No & No \\
\hline Saving search queries & No & Yes & No \\
\hline RSS Feed for search queries & Yes & Yes & No \\
\hline Search history & Yes & Yes & No \\
\hline
\end{tabular}

Table 4: Other Search Features

\subsection{Result List and Bibliographic View}

Once a search query is introduced, another important aspect to consider is how the database system displays the results to the user and what options and useful features it provides. We compared several aspects as detailed in Table 5. First of all, we wanted to know if the fields displayed in the result list are configurable, i.e. if the user can select the fields they want to be displayed in the result list. This was the case for Patentscope and Depatisnet but not for Espacenet, whereas the possibility of field sorting was provided by all three search systems. Unfortunately, the ability to filter the results by certain criteria (i.e. publication dates or applicants) - which is a very useful feature that exists in most commercial providers - was not available on the three search systems compared in this study.

Keyword highlighting in the results is, in our experience, another highly useful feature, which both Espacenet and Patentscope support, but which Depatisnet does not. Regarding the presentation of miniature images 
(thumbnails) in the result list, only Patentscope provides the functionality, which is a powerful feature since it can help a patent searcher perform a quicker screen of the results when dealing with a larger patent result list.

Another aspect compared in this study was the maximum number of results the search system was able to display, which is an important issue when large amounts of patent publications need to be retrieved for further processing. In this case only Patentscope showed to have no number limitation, whereas Depatisnet has a limitation of maximum 1,000 results and Espacenet 500 results.

\begin{tabular}{|c|c|c|c|}
\hline & ESPACENET & PATENTSCOPE & DEPATISNET \\
\hline Configurable fields in view & No & Yes & Yes \\
\hline Sorting & Yes & Yes & Yes \\
\hline Results filtering & No & No & No \\
\hline Keyword highlighting & Yes & Yes & No \\
\hline Image thumbnails & No & Yes & No \\
\hline Family grouping & Yes & Yes & Yes (not by default) \\
\hline Maximum number of results & 500 & unlimited & 1,000 \\
\hline
\end{tabular}

Table 5: Result List Features

The next logical step after comparing the results list was to compare the bibliographic view of the patents and what features each system provided here. As can be seen in Table 6, Espacenet proved to be the most complete solution, offering patent legal status information (via Inpadoc legal status database) and direct linking to citing and cited documents of the patent publication, which can be very useful for a patent searcher and was, in our opinion, very beneficial when comparing it to the other two search systems.

\begin{tabular}{|c|c|c|c|}
\hline & ESPACENET & PATENTSCOPE & DEPATISNET \\
\hline Link to citing documents & Yes & No & No \\
\hline Link to cited documents & Yes & No & No \\
\hline Link to legal status & Yes (INPADOC) & No & $\begin{array}{c}\text { Yes (DE documents } \\
\text { only) }\end{array}$ \\
\hline Image thumbnails & Yes & Yes & No \\
\hline Link to original document & Yes & Yes & Yes \\
\hline Full document download & $\begin{array}{l}\text { Yes (with } \\
\text { CAPTCHA) }\end{array}$ & Yes & $\begin{array}{l}\text { Yes (with } \\
\text { CAPTCHA) }\end{array}$ \\
\hline Patent machine translation & Yes (Google) & $\begin{array}{c}\text { Yes (Google, Bing, } \\
\text { Tapta) }\end{array}$ & No \\
\hline
\end{tabular}

Table 6: Bibliographic View Features

Another feature we compared was the possibility of automatically translating patent publications into other languages. Depatisnet offered no patent translation engine, whereas both Espacenet and Patentscope offered integrated machine translation which can translate patent publications into several languages. In both cases the technology comes from Google- with Patentscope also offering an alternative translation engine from Microsoft. Patentscope additionally has a new translating tool named TAPTA, which can be an interesting alternative for difficult translations, since it is specifically built to translate titles and abstracts and can be adapted to a technical domain[15].This means the translation will take into account specific vocabulary according to the technical field of the translated patent.

\subsection{Patent Data Export}

The last aspect of our comparison was the data export functionality, where we analysed the manner in which patent data could be saved for further processing (Table 7). 


\begin{tabular}{|c|c|c|c|}
\hline & ESPACENET & PATENTSCOPE & DEPATISNET \\
\hline $\begin{array}{r}\mathrm{Nr} \text { of max. exportable patents } \\
\text { records }\end{array}$ & 500 & $\begin{array}{l}10,000 \text { (with priority } \\
\text { data) / } 100 \text { (with } \\
\text { images \& abstract) }\end{array}$ & 1,000 \\
\hline $\mathrm{Nr}$ of max. fields in export & 24 & 8 & 9 \\
\hline Format & CSV, XLS & XLS & CSV, XLS \\
\hline With images & No & Yes & No \\
\hline With abstract & Yes & Yes & No \\
\hline
\end{tabular}

Table 7: Data Export Features

In this comparison Patentscope was, in our opinion, the best of the compared systems, mainly for two reasons. First, Patentscope was the only system that also offered exporting images, which is a very useful feature when you want to export smaller lists for future integration in patent reports. The second reason is the high number of exportable patent records allowed, which is highly beneficial when a user wants to use the data for further statistical analysis. Patentscope has a new export function which allows exporting up to 10,000 patent records, which is considerably more than the maximum of 500 with Espacenet and the maximum of 1,000 records with Depatisnet. On the other hand, Espacenet showed to be the search system that had the highest numbers of exportable fields, with 24 fields, versus 8 and 9 in Patentscope and Depatisnet respectively.

\subsection{Unique Features}

When comparing the three patent search systems we also found some unique features, which none of the other patent search systems had.

One example is the "My Patents List" feature in Espacenet (Fig. 9). This feature, known from other database systems as 'marked list' or 'favourite list' lets the user mark patents, which are then saved in a separate list, independent of the search being performed. This feature can be very helpful, especially when conducting iterative searches. One aspect to take into account is that Espacenet only saves this list locally, which means that the marked patents are only saved on the computer the user is working from and not on a server.

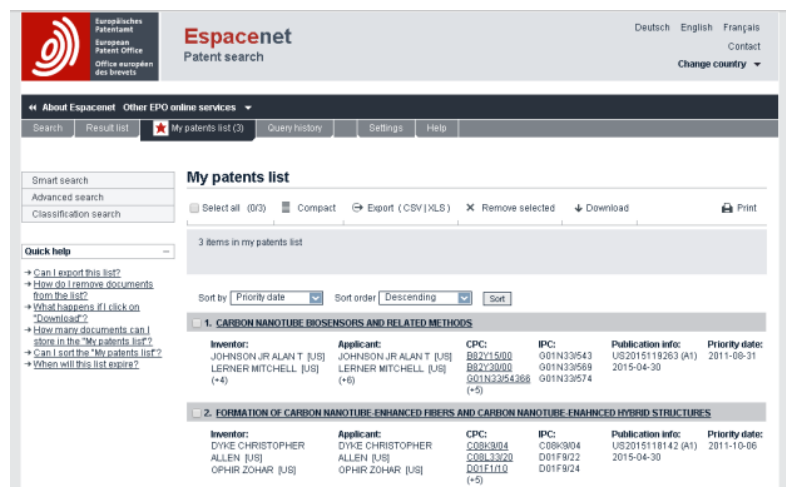

Fig. 9: "My Patents List" (Screenshot from Espacenet)

The unique standout feature of Patentscope is, without doubt, the functionality which allows the user to obtain a statistical analysis of the results since this is normally a feature only commercial providers offer (Fig. 10). Patentscope lets you generate a ranking of top patent offices, main ipc, main applicant, main inventor and publication date, either displayed in bar or pie charts. Naturally, when compared to commercial providers, the configuration possibilities and the visualization options are limited. Patentscope does not, for example, allow you to configure the variables of the analysis, and the applicant data is not harmonized. Nevertheless this remains a very useful feature, especially when compared to the other two free of charge database systems. 


\section{Conclusion}

As we have seen in the comparison study Espacenet and Depatisnet have the best data coverage, although Patentscope has the best full text searching capability.

Regarding the database features and functionalities we can summarize the following outline:

\section{Espacenet}

Advantages

- $\quad$ CPC searchable and features integration in search interface

- Marked patent list ("My Patents List")

- $\quad$ Links to cited \& citing documents and legal status

Disadvantages

- Term number limitations in the search interface

Patentscope

Advantages

- Image thumbnails in result lists

- Best searchable full text coverage

- Basic statistical analysis and patent data export capability

Disadvantages

- Number search engine

\section{Depatisnet}

Advantages

- Most versatile number search

- Left truncation possible

- Ikofax search mode for complex queries

Disadvantages

- No keyword highlighting and no images in bibliographic view 
We have therefore concluded that of the three compared search tools:

- $\quad$ Espacenet-> best features for searching

- Patentscope-> best for analysis

- Depatisnet-> best for complex search tasks

Nevertheless we would like to see the following features implemented in the near future which would bring the patent search experience of the compared free of cost products to a new level:

- Image thumbnails in result list within Espacenet and Depatisnet

- Statistical analysis for Espacenet and Depatisnet

- Mobile versions adapted for touch screens on smartphones and tablets

- Possibility to filter the result list

- Possibility to combine steps of search history

\section{Acknowledgements}

This article is based on a presentation given at the European Patent Office Patent Information Conference (EPOPIC) that was held during 4-6 November 2014 in Warsaw, Poland. Special thanks go to the organization team of the conference from the European Patent Office and to the CITPIA Patlib Centre Team without whom this study would never have been possible.

The authors acknowledge the support of Spanish Ministry of Science and Economy for funding the framework project "Vigilancia tecnologica de la nanotecnologia espanola a traves de sus patentes (Technology watch of Spanish Nanotechnology via its patents)", grant by Plan Nacional de I+D+i 2008-2011, project code: CSO201238801, for which this study was used.

\section{References}

[1] Smith, D. (1988), A comparison of some patent databases. World Patent Information, 1988, vol. 10, issue 1, pages $11-16$

[2] Lambert, N (1999), Patents on the Internet versus patents online: A snapshot in time. Journal of Chemical Information and Computer Sciences, Vol. 39, pp. 448-452.

[3] Schwander, P. (2000), An evaluation of patent searching resources: comparing the professional and free online databases. World Patent Information, 2000, vol. 22, issue 3, pages 147-165

[4] Stock, M. and Stock, W. G. (2006), Intellectual property information: A comparative analysis of main information providers. Journal of the American Society for Information Science and Technology, Vol. 57, pp. 1794-1803.

[5] González-Albo Manglano B, Zulueta, M (2007), Estudio comparativo de bases de datos de patentes en internet (Internet patent databases: a comparative study). Anales de Documentación. University of Murcia, Spain. pp.145-162.

[6] European Patent Office - Information on EPO data - Useful tables and statistics http://www.epo.org/searching/data/data/tables/weekly.htmlAccessed: 23.09.2014

[7]World Intellectual Property Organization - Patentscope - National Collections - Data Coverage http://patentscope.wipo.int/search/en/help/data_coverage.jsf Accessed: 23.09.2014

[8]Deutsches Patent- und Markenamt - DEPATISnet- Datenbestand https://depatisnet.dpma.de/DepatisNet/depatisnet?action=datenbestand Accessed: 23.09.2014 
[9] Ammann S. Personal communication, 20.10.2014, Patent Information Team, World Intellectual Property Organization.

[10] Diaconescu, I. (2014) WIPO - patent systems news! - Presentation at EPO - Patent information conference 2014 held at 4-6 November 2014 in Warsaw, Poland, pp. 4, World Intellectual Property Organization

[11 European Patent Office - Espacenet - Help - Smart search- Entering Queries http://worldwide.espacenet.com/help?locale=en_EP\&method=handleHelpTopic\&topic=searchquery

[12] World Intellectual Property Organization - Patentscope - National Collections - Field Definitions http://patentscope.wipo.int/search/en/help/fieldsHelp.jsf Accessed: 28.09.2014

[13] Deutsches Patent- und Markenamt - DEPATISnet Hilfe - Recherchierbare Felder https://depatisnet.dpma.de/depatisnet/htdocs/prod/de/hilfe/recherchierbarefelder/index.html Accessed: 28.09.2014

[14] European Patent Office - Espacenet - Help - Smart search - field identifiers http://worldwide.espacenet.com/help?locale=en_EP\&method=handleHelpTopic\&topic=fieldidentifier Accessed: 28.09.2014

[15] Translation Assistant for Patent Titles and Abstracts (TAPTA) https://www3.wipo.int/patentscope/translate/translate.jsf?interfaceLanguage=en Accessed: 28.09.2014

[16] Deutsches Patent- und Markenamt - DEPATISnet Hilfe - IKOFAX Search mode https://depatisnet.dpma.de/prod/en/hilfe/recherchemodi/ikofax-recherche/index.html Accessed: 28.09.2014 